\title{
Utility of Follow-up With Absolute Lymphocyte Count in Patients Undergoing Eribulin Treatment for Early Detection of Progressive Advanced or Metastatic Breast Cancer
}

\author{
WATARU GOTO ${ }^{1}$, SHINICHIRO KASHIWAGI ${ }^{1}$, KOJI TAKADA ${ }^{1}$, YUKA ASANO $^{1}$, TAMAMI MORISAKI ${ }^{1}$, \\ MASATSUNE SHIBUTANI ${ }^{2}$, HIROAKI TANAKA $^{2}$, KOSEI HIRAKAWA $^{1,2}$ and MASAICHI OHIRA ${ }^{1,2}$ \\ ${ }^{1}$ Department of Breast and Endocrine Surgery, Osaka City University Graduate School of Medicine, Osaka, Japan; \\ ${ }^{2}$ Department of Gastroenterological Surgery, Osaka City University Graduate School of Medicine, Osaka, Japan
}

\begin{abstract}
Background/Aim: Eribulin, a non-taxane microtubule inhibitor, improves the tumor immune microenvironment via vascular remodeling. Systemic peripheral immune markers such as absolute lymphocyte count (ALC) and neutrophil-tolymphocyte ratio (NLR) have been reported as prognostic factors of patients treated with eribulin. However, the usefulness of the longitudinal changes of these blood parameters during eribulin treatment remains unknown. Patients and Methods: A total of 97 patients with locally advanced or metastatic breast cancer treated with eribulin were recruited. ALC and NLR values were collected at each cycle of treatment. The correlation between ALC and NLR and prognosis and antitumor effects was retrospectively evaluated. Results: Progression-free and overall survival for patients with a base line $A L C \geq 1,500 / \mu l$ was significantly better than that for those with a lower $A L C$ ( $p=0.049$ and $p=0.004$, respectively). The mean ALC in the nonprogressive disease $(P D)$ group increased, while that in the $P D$ group slightly decreased over time. A low ALC at the last cycle was significantly correlated with PD $(p=0.030)$. Of the 64 patients with $P D, 47$ were classified as having progression due to a pre-existing lesion (PPL), and 17 patients were classified as having progression due to new metastasis (PNM). The mean $A L C$ for the PPL group slightly increased during eribulin treatment, while that for the PNM group decreased. Conclusion:
\end{abstract}

This article is freely accessible online.

Correspondence to: Shinichiro Kashiwagi, MD, Ph.D., Department of Breast and Endocrine Surgery, Osaka City University Graduate School of Medicine, 1-4-3 Asahi-machi, Abeno-ku, Osaka 5458585, Japan. Tel: +81 666453838, Fax: +81 666466450, e-mail: kashiwa@med.osaka-cu.ac.jp

Key Words: Eribulin, absolute lymphocyte count, progression-free survival, advanced or metastatic breast cancer, tumor immunology.
Eribulin treatment may have improved the immune status in eribulin responders. Monitoring ALC values may be useful for early assessment of response to therapy.

Eribulin, a non-taxane, synthetic inhibitor of microtubule dynamics, is currently an approved treatment for locally advanced or metastatic breast cancer (MBC). It induces $\mathrm{G}_{2} / \mathrm{M}$ cell-cycle arrest and subsequent apoptosis (1-3). Eribulin also has some unique antineoplastic effects in breast cancer, such as improvement of tumor perfusion, hypoxia (4), and epithelial-mesenchymal transition $(5,6)$. Moreover, eribulin enhances the antitumor immune response (7), and tumor-infiltrating lymphocytes (TILs) may be a good predictor of the therapeutic effect eribulin in patients with triple-negative breast cancer (8).

In addition to TILs, systemic peripheral immune markers have been shown to be significant prognostic factors in patients with MBC treated with eribulin. Miyagawa et al. reported that a baseline neutrophil-to-lymphocyte ratio (NLR) <3 was significantly associated with good progression-free survival (PFS) (9). Miyoshi et al. also showed that eribulin prolonged overall survival (OS) compared to treatment of physician's choice in patients with a baseline absolute lymphocyte count (ALC) $\geq 1,500 / \mu 1$ (10). These studies suggest that the status of the tumor immune microenvironment affects the efficacy of eribulin.

However, progressive disease (PD) can occur even in patients with a good baseline ALC or NLR. Eribulin was shown to enhance the antitumor immune activity in responders (7); thus, it was hypothesized that real-time monitoring of these blood parameters in patients treated with eribulin can predict PD and is useful in guiding treatment. This study aimed to explore the longitudinal changes in ALC and NLR of patients with MBC undergoing eribulin treatment and to identify whether these systemic peripheral markers are useful predictors of PD. 


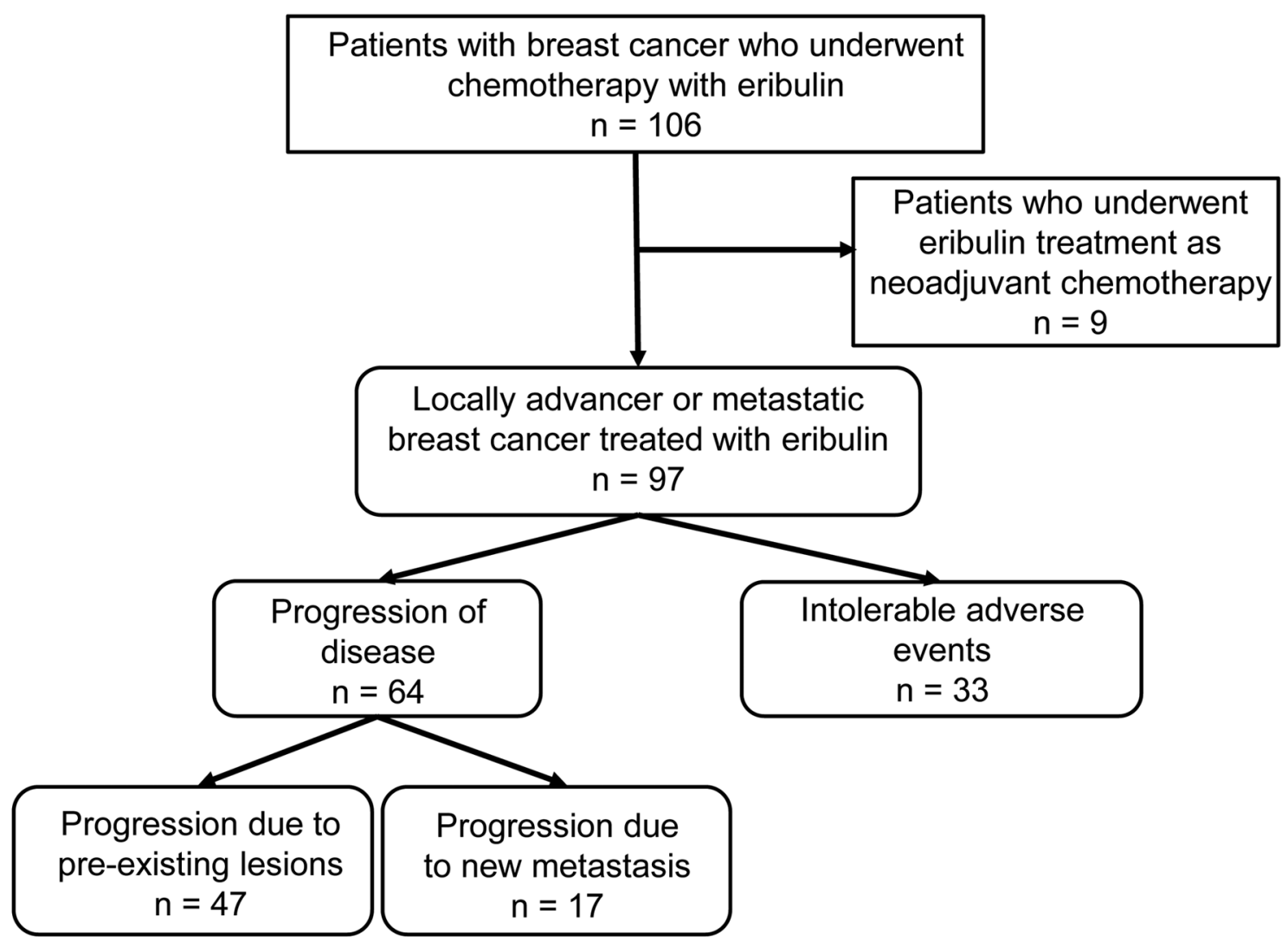

Figure 1. Consort diagram. A total of 106 patients with breast cancer underwent eribulin treatment from August 2011 to April 2019. In the present study, nine patients treated with neoadjuvant chemotherapy were excluded, and 97 patients were included.

\section{Patients and Methods}

Patient background. This study was conducted at Osaka City University Graduate School of Medicine (Osaka, Japan) and conformed to the provisions of the Declaration of Helsinki of the World Medical Association. The study protocol was approved by the Ethics Committee of Osaka City University (\#926). At the time of treatment, written informed consent was obtained from all patients. The electronic medical data of patients with breast cancer treated with eribulin from August 2011 to April 2019 at the Osaka City University Graduate School of Medicine (Osaka, Japan) were obtained and analyzed. A total of 106 patients were identified from their medical records. Nine patients who underwent eribulin treatment as neoadjuvant chemotherapy were excluded, and 97 patients with $\mathrm{MBC}$ were included. $\mathrm{T}$ and $\mathrm{N}$ factors and tumor stage were stratified based on the seventh edition of the Union for International Cancer Control TNM classification (11). Tumors were classified into intrinsic subtypes according to the immunohistochemical expression of estrogen receptor, progesterone receptor, and human epidermal growth factor receptor 2; the hormone-positive group comprised patients with estrogen- or progesterone-positive patients.

Based on the chemotherapy regimen, eribulin mesylate $\left(1.4 \mathrm{mg} / \mathrm{m}^{2}\right)$ was administered intravenously on days 1 and 8 in 21-day cycles (12, 13). For patients with a reduced neutrophil count, treatment was delayed. Administration was repeated until PD was detected or intolerable adverse events requiring the discontinuation of the scheduled chemotherapy were noted. All patients received this protocol at the outpatient clinic. The antitumor effect was evaluated based on the Response Evaluation Criteria in Solid Tumors version 1.1 (14). PD was defined as progression due to a pre-existing lesion (PPL)/due to new metastasis (PNM) based on systemic computed tomography findings (Figure 1). OS was defined as the time from the start of eribulin treatment to death from any cause. PFS was defined as the time from the start to the end of treatment because of PD or death from any cause. The median follow-up time for the assessment of OS was 494 days (range=7-2,955 days) and for PFS was 105 days (range $=7-818$ days).

Blood sample analysis. Blood samples were obtained at each cycle before eribulin treatment, and the differential white blood cell counts were analyzed using a Coulter LH 750 Hematology Analyzer (Beckman Coulter, Brea, CA, USA). The NLR was calculated from the blood sample by dividing the absolute neutrophil count by the ALC.

Statistical analysis. Statistical analysis was performed using the JMP13 software program (SAS Institute, Cary, NC, USA). Associations among variables were analyzed using chi-squared or Fisher's exact test, as appropriate. OS and PFS were estimated using the Kaplan-Meier method and compared between groups using the 
A

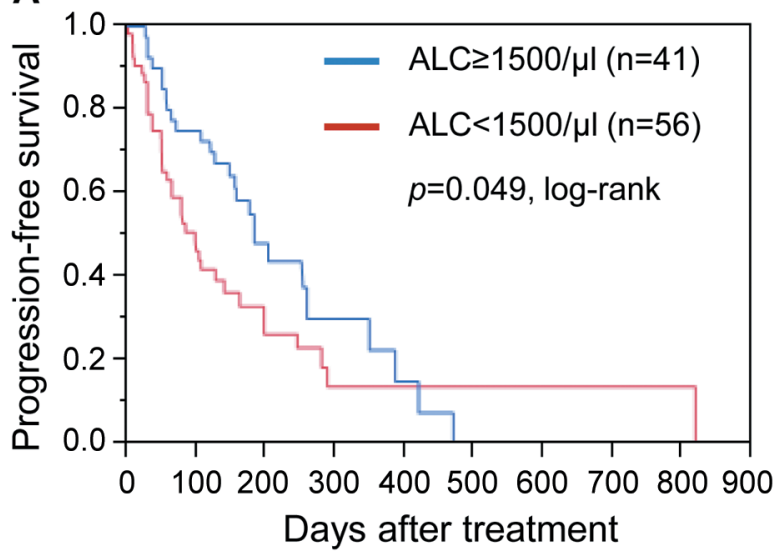

C

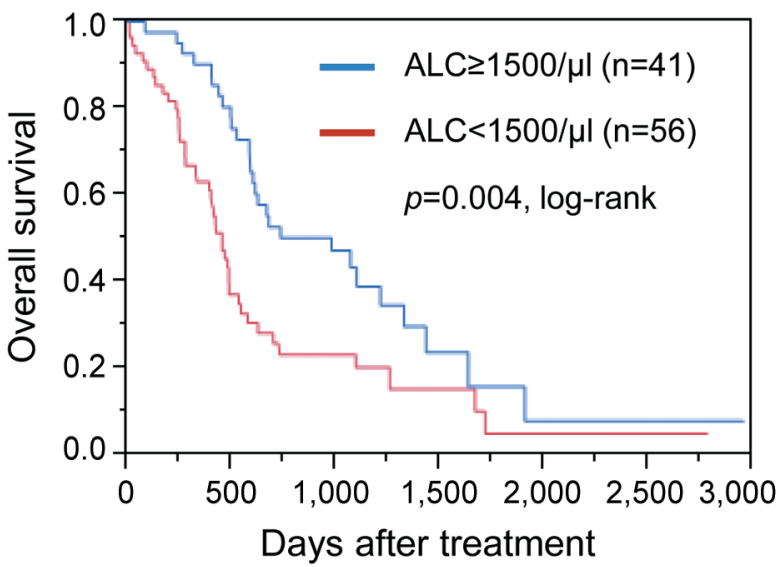

B

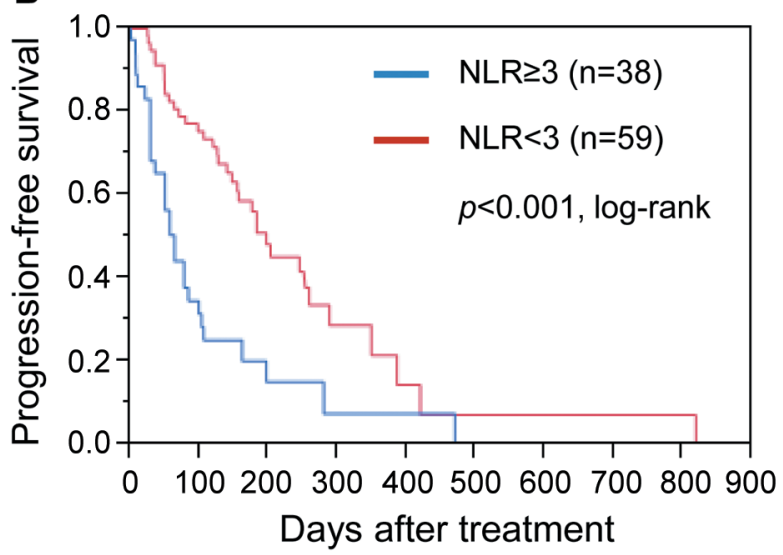

D

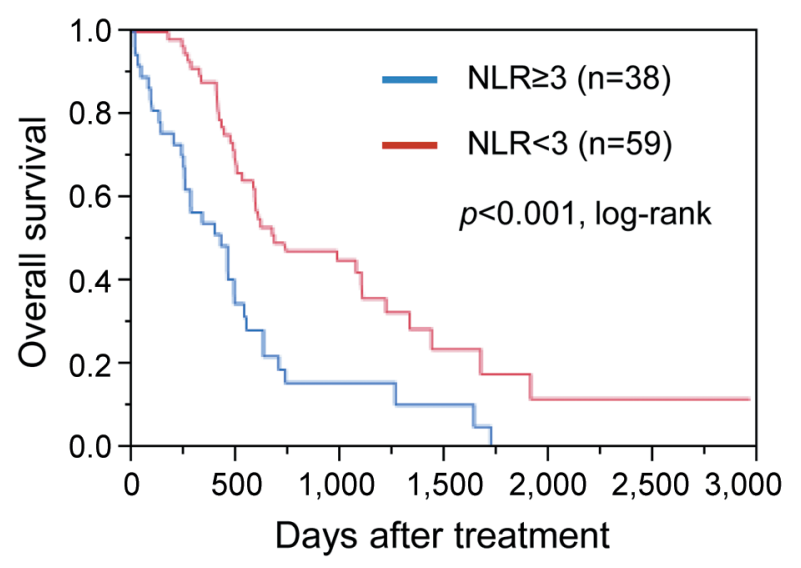

Figure 2. Survival was analyzed according to absolute lymphocyte count (ALC) and neutrophil-to-lymphocyte ratio (NLR). A: Progression-free survival was significantly worse in the group with low ALC compared with the high-ALC group $(p=0.049)$. B: PFS was significantly worse in the group with high NLR compared with the low-NLR group $(p<0.001)$. C: Overall survival was also significantly worse in the low-ALC group compared with the high-ALC group $(p=0.004)$. D: Overall survival was significantly worse in the group with a high NLR compared with the low-NLR group ( $p<0.001)$.

log-rank test. To select the most appropriate cut-off value for ALC and NLR, receiver operating characteristic curve analysis was conducted. A p-value of less than 0.05 was considered significant.

\section{Results}

Relationship between ALC and NLR at baseline and patient prognosis. As previously reported, using a cut-off of $1,500 / \mu \mathrm{l}$ for ALC and of 3 for NLR, the relationship between ALC and NLR at the baseline and patient prognosis $(9,10)$ were examined. PFS for patients with a baseline ALC $\geq 1,500 / \mu 1$ was significantly better than that for those with a lower ALC $(p=0.049)$. Furthermore, PFS for patients with a baseline NLR $<3$ was significantly better than that for those with a higher NLR $(p<0.001)$ (Figure 2A and B). Similar to PFS, OS for patients with a baseline ALC $\geq 1,500 / \mu 1$ was significantly better than that for those with a lower ALC $(p=0.004)$. Moreover, OS for patients with an NLR at the baseline $<3$ was significantly better than that for those with a higher NLR $(p<0.001)$ (Figure 2C and D).

Clinicopathological features and outcome of eribulin treatment. In the present study, all patients were classified into a PD $(n=64,66.0 \%)$ or non-PD $(n=33,34.0 \%)$ group, and the relationship between clinicopathological features and treatment effect of eribulin are shown in Table I. PD tended to be observed more frequently in patients with human epidermal growth factor receptor 2-enriched breast cancer and triple-negative breast cancer $(p=0.050)$. Moreover, the response to eribulin was significantly worse in patients with de novo advanced breast cancer $(p=0.005)$. There was no significant correlation between outcome of eribulin and any other tested clinicopathological parameter, including number of previous chemotherapy regimens. 
Table I. Correlation between clinicopathological features and treatment effect.

\begin{tabular}{|c|c|c|c|}
\hline \multirow[t]{2}{*}{ Parameter } & \multicolumn{2}{|c|}{ Treatment effect, n (\%) } & \multirow[t]{2}{*}{$p$-Value } \\
\hline & $\begin{array}{c}\mathrm{PD}(\mathrm{n}=64 \\
66.0 \%)\end{array}$ & $\begin{array}{c}\text { Non-PD }(\mathrm{n}=33, \\
34.0 \%)\end{array}$ & \\
\hline \multicolumn{4}{|l|}{ Age at operation } \\
\hline$<60$ Years & $30(46.9 \%)$ & $17(51.5 \%)$ & \\
\hline$\geq 60$ Years & $34(53.3 \%)$ & $16(48.5 \%)$ & 0.675 \\
\hline \multicolumn{4}{|l|}{ Subtype } \\
\hline Luminal & $29(45.3 \%)$ & $19(57.6 \%)$ & \\
\hline Luminal-HER & $1(1.6 \%)$ & $4(12.1 \%)$ & \\
\hline HER2-enriched & $4(6.3 \%)$ & $1(3.0 \%)$ & \\
\hline TNBC & $30(46.8 \%)$ & $9(27.3 \%)$ & 0.050 \\
\hline \multicolumn{4}{|c|}{ Previous CHT regimens } \\
\hline$\leq 3$ & $27(42.2 \%)$ & $11(33.3 \%)$ & \\
\hline$>3$ & $37(57.8 \%)$ & $22(66.7 \%)$ & 0.511 \\
\hline \multicolumn{4}{|l|}{ Metastatic site } \\
\hline Visceral & $47(73.4 \%)$ & $19(57.6 \%)$ & \\
\hline Non-visceral & $17(26.6 \%)$ & $14(42.4 \%)$ & 0.167 \\
\hline \multicolumn{4}{|l|}{$\begin{array}{l}\text { Tumor status at } \\
\text { eribulin treatment }\end{array}$} \\
\hline De novo & $13(20.3 \%)$ & $16(48.5 \%)$ & \\
\hline Recurrence & $51(79.7 \%)$ & $17(51.5 \%)$ & 0.005 \\
\hline
\end{tabular}

CHT: Chemotherapy; HER2: human epidermal growth factor receptor 2; TNBC: triple-negative breast cancer; PD: progressive disease.

Evaluation before and after eribulin treatment. Pre- and postdose evaluations of ALC and NLR were performed according to the values recorded at the first and last cycle of eribulin treatment. Longitudinal courses of ALC and NLR for the PD and non-PD groups are shown in Figure 3. The mean ALC at the first cycle was lower for the PD group than for the nonPD group $(1,423 / \mu l v s .1,538 / \mu l)$. In addition, the mean value of ALC at the last cycle in the PD group was slightly lower than that at the first cycle $(1,414 / \mu \mathrm{l})$, while that for the nonPD group tended to increase during treatment $(1,643 / \mu \mathrm{l})$ (Figure 3A). Regarding NLR, the PD group had higher mean values at the first cycle than the non-PD group (4.03 vs. 2.75). Interestingly, unlike ALC, the mean NLR decreased in both groups at the last cycle of eribulin treatment $(4.03 \rightarrow 3.44$ and $2.75 \rightarrow 2.13$, respectively) (Figure 3B).

The ALC cut-off value for PD at the last cycle was $1,318 / \mu 1$ (area under the receiver operating characteristics curve $=0.60$; sensitivity $=51.6 \%$; specificity $=69.7 \%$ ) (Figure 4A). Patients were then classified into low-ALC and highALC groups according to this cut-off value and a low ALC at the last cycle was significantly associated with PD $(p=0.030)$. The NLR cut-off value for PD at the last cycle was 1.66 (area under the receiver operating characteristics curve $=0.55$; sensitivity $=60.9 \%$; specificity $=54.6 \%$ ) (Figure 4B). However, there was no significant relationship between NLR values at the last cycle and PD ( $p=0.196)$ (Table II).
Peripheral immune markers and differences in progression type. Of the 64 patients with observed PD, there were significantly more patients in the PPL group $(n=47,73.4 \%)$ than in the PNM group $(n=17,16.6 \%)$. A longitudinal analysis of ALC and NLR was also performed according to PPL and PNM. The mean value of ALC at the first cycle was lower in the PPL group than in the PNM group $(1,354 / \mu l v s$. $1,616 / \mu \mathrm{l})$. Interestingly, the mean ALC in the PPL group slightly increased during eribulin treatment (to $1,378 / \mu \mathrm{l}$ ), while that for the PNM group decreased $(1,516 / \mu 1)$ (Figure 5A). Regarding NLR, the PPL group had a higher mean value at the first cycle than did the PNM group (4.37 vs. 3.10 ). In addition, the mean value of NLR decreased in both groups (to 3.77 and 2.51, respectively) (Figure 5B).

\section{Discussion}

In this study, the utility of follow-up using the systemic peripheral immune markers ALC and NLR in patients with MBC undergoing eribulin treatment was verified. Lymphocytes play an important role in host tumor immunity by causong cytotoxic cell death or inhibition of tumor cell proliferation (15-17). In contrast, neutrophils reflect cancerassociated inflammation, which promotes tumor cell proliferation and distant metastasis (18). Therefore, it was considered that the ALC seems to be a more sensitive indicator of the immune status of patients with cancer than the NLR. Throughout eribulin treatment, the mean ALC for the PD group was lower than that for the non-PD group, whilst the mean NLR was higher. This suggests that eribulin may be less effective in MBC with low systemic immune activity. In addition, eribulin treatment reduced the NLR in both groups, while the ALC increased only in the non-PD group. It is likely that ALC is a better marker than NLR in reflecting the therapeutic effect of eribulin. Furthermore, some previous studies have reported that eribulin improves the local tumor immune microenvironment $(4,5,7)$; however, in this longitudinal analysis, increased ALC and reduced NLR in the non-PD group suggests that eribulin has the ability to enhance not only the local immune status but also the systemic immune status.

In the present study, an ALC at the last cycle $<1,318 / \mu 1$ was significantly associated with PD, while the NLR at the last cycle was not a useful predictive marker of PD. The measurement of serum tumor makers such as carcinoembryonic antigen and cancer antigen 15-3 has been considered an economic and less invasive examination in clinical practice; however, whether monitoring these markers during treatment in patients with $\mathrm{MBC}$ is useful for assessing response to therapy is controversial (19-22). The results suggest that in patients with MBC, follow-up using ALC during eribulin treatment may be useful to evaluate the antitumor effect and determine when to stop administration of eribulin. 
A

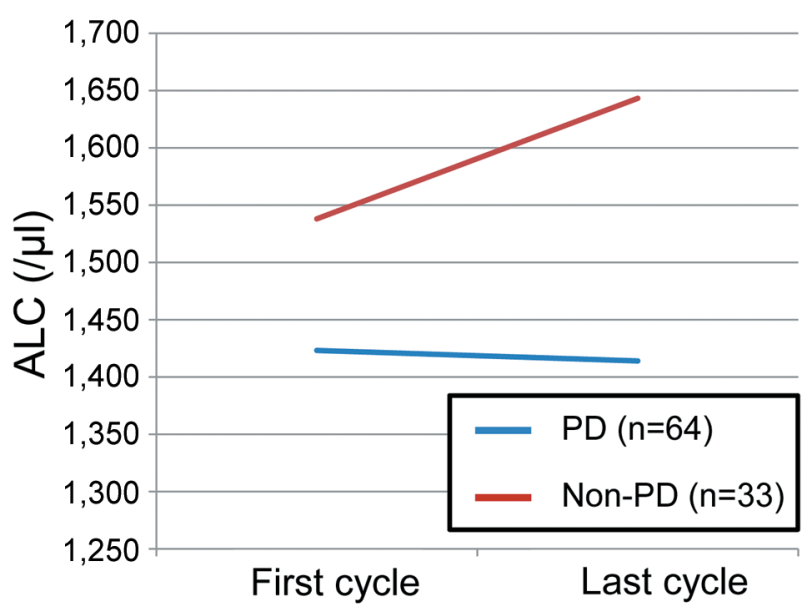

B

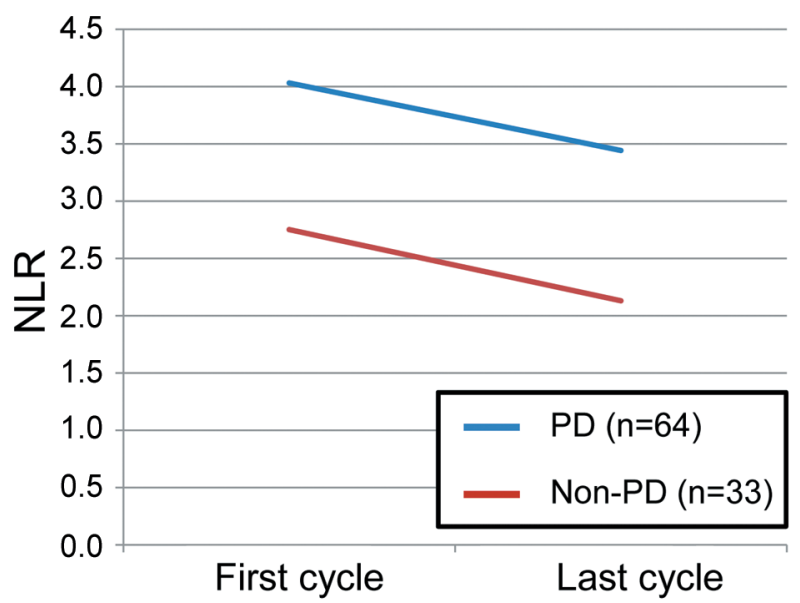

Figure 3. Mean absolute lymphocyte count $(A L C)(A)$ and neutrophil-to-lymphocyte ratio $(N L R)(B)$ at the first and last cycles of eribulin treatment according to progressive disease (PD). The mean ALC for the non-PD group increased, while that of the PD group was slightly reduced. Unlike $A L C$, the mean NLR at the last cycle was lower than at the first cycle for both groups.

A

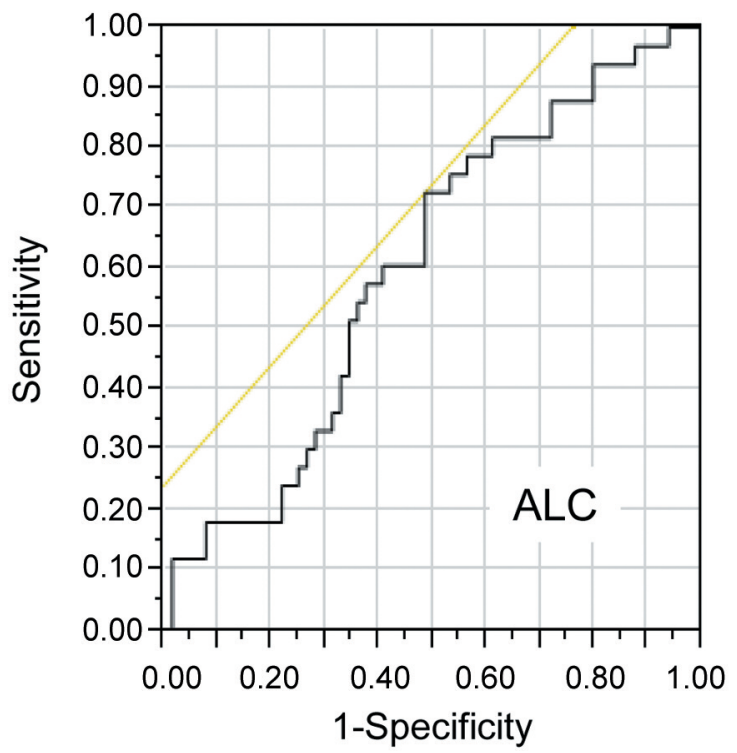

B

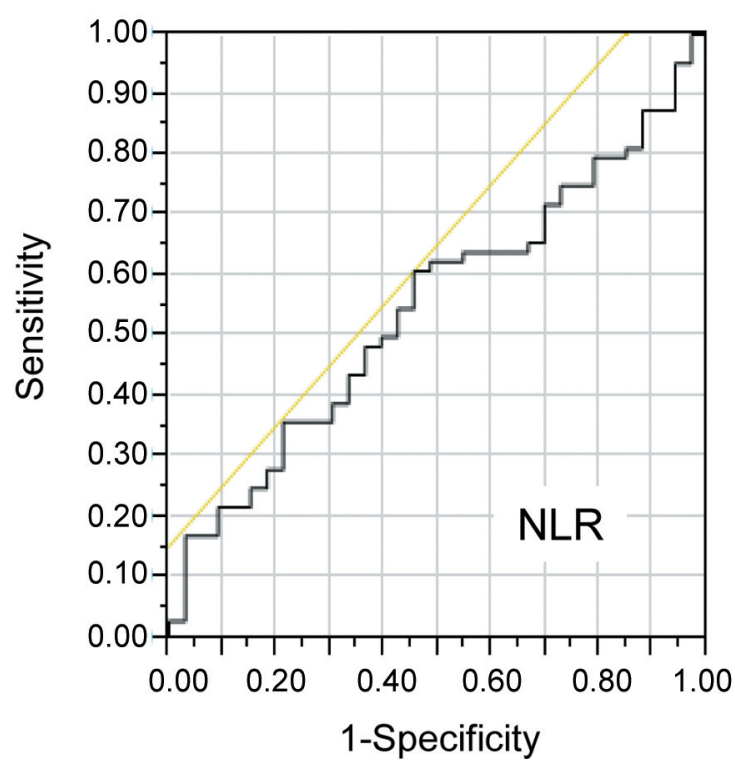

Figure 4. Receiver operating characteristics curve analyses for prediction of progressive disease (PD) by the absolute lymphocyte count $(A L C)(A)$ and neutrophil-to-lymphocyte ratio $(N L R)(B)$ at the last cycle of eribulin treatment of patients with metastatic breast cancer. The ALC cut-off at the last cycle for PD was 1,318/ $\mu$ l (area under the curve=0.60; sensitivity=51.6\%; specificity=69.7\%). The NLR cut-off at the last cycle for PD was 1.66 (area under the curve $=0.55$; sensitivity $=60.9 \%$; specificity $=54.6 \%$ ).

Patients with observed PD were also stratified into PPL and PNM groups. Eribulin treatment reduced the ALC only in the PNM group. It seems that a decrease in immune activity during eribulin treatment may induce migration of breast cancer cells.
The response to eribulin is significantly associated with improvement of immune activity (7); therefore, a decrease in ALC (deterioration of immunity) may mean that eribulin was ineffective, and suppression of epithelial-mesenchymal 
A

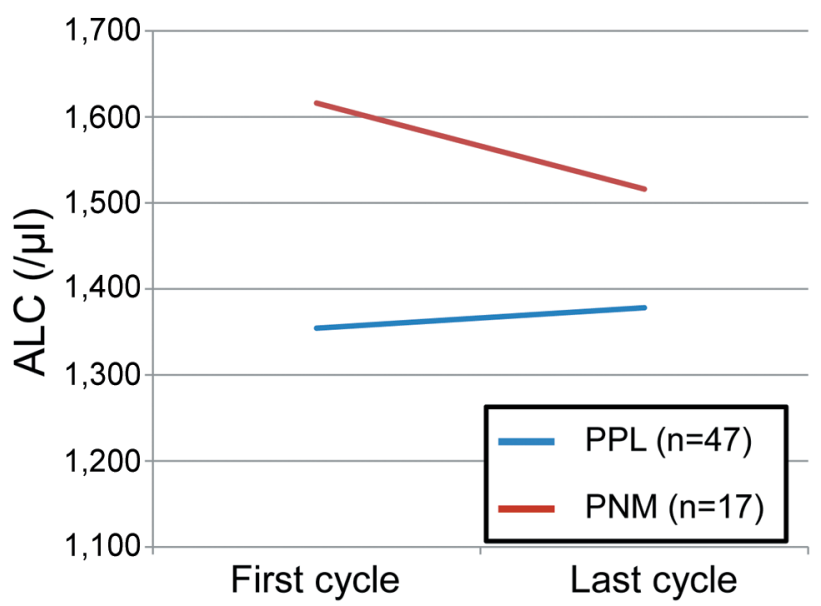

B

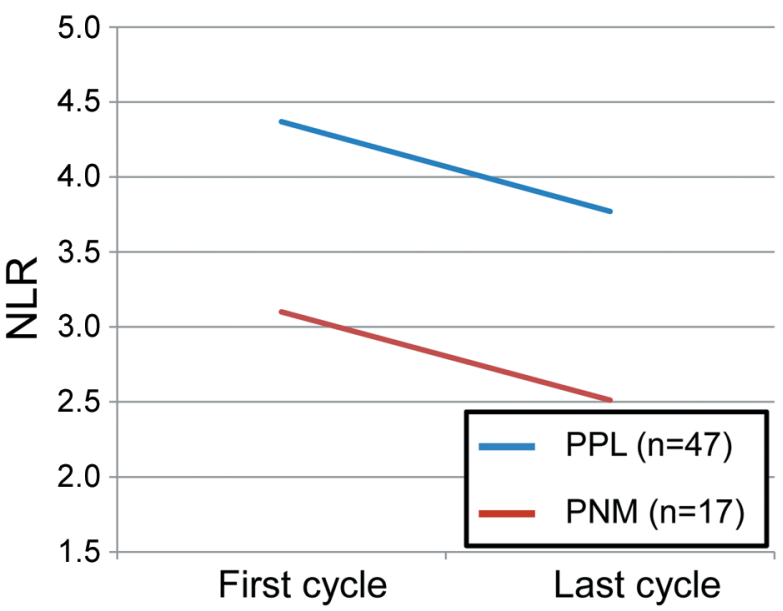

Figure 5. Mean absolute lymphocyte count $(A L C)(A)$ and neutrophil-to-lymphocyte ratio $(N L R)(B)$ at the last cycle of eribulin treatment in patients with progression from a pre-existing lesion (PPL) or progression due to new metastasis (PNM). The mean ALC for the PPL group slightly increased during eribulin treatment, while that for the PNM group decreased. Unlike ALC, the mean NLR decreased for both groups.

transition may also be inhibited. It was previously reported that the progression of cancer in patients undergoing eribulin treatment was significantly associated with the state of the immune tumor microenvironment as evaluated through TILs (23). Unlike TILs of biopsy specimens, the measurement of ALC, a peripheral systemic immune marker, is a simpler and less invasive examination.

This study has a few limitations. Firstly, this was a singlecenter retrospective study; hence, the sample size was small. Secondly, each ALC value was calculated only when the criteria for eribulin administration were met; however, the side-effects of eribulin might have reduced the ALC.

Our findings suggest that monitoring ALC in patients with MBC undergoing eribulin therapy is important in evaluating treatment efficacy and tumor progression. Thus, further prospective multicenter studies are needed to identify the strengths and weaknesses of these findings.

\section{Conclusion}

This is the first study to demonstrate the longitudinal changes in ALC and NLR during eribulin treatment in patients with MBC. ALC can easily be measured in daily clinical practice during treatment and is useful in evaluating the immune status. The findings of this study indicate that follow-up with ALC evaluation may be useful for assessing response to therapy and planning treatment strategies for MBC.

\section{Conflicts of Interest}

All of the Authors have no conflicts of interest to disclose regarding this study.
Table II. Correlation of absolute lymphocyte count (ALC) and neutrophil to lymphocyte ratio (NLR) at the last cycle with treatment effect.

\begin{tabular}{lllll}
\hline Parameters & & \multicolumn{2}{c}{ Treatment effect } & \multirow{2}{*}{-Value } \\
\cline { 3 - 4 } & Cut-off & $\begin{array}{c}\text { PD }(\mathrm{n}=64, \\
66.0 \%)\end{array}$ & $\begin{array}{c}\text { Non-PD }(\mathrm{n}=33, \\
34.0 \%)\end{array}$ & \\
& & & & \\
ALC at last cycle & $<1,318 / \mu \mathrm{l}$ & $33(51.6 \%)$ & $9(27.3 \%)$ & \\
& $\geq 1,318 / \mu \mathrm{l}$ & $31(48.4 \%)$ & $24(72.7 \%)$ & 0.030 \\
NLR at last cycle & $<1.66$ & $25(39.1 \%)$ & $18(54.5 \%)$ & \\
& $\geq 1.66$ & $39(60.9 \%)$ & $15(45.5 \%)$ & 0.196
\end{tabular}

PD: Progressive disease.

\section{Authors' Contributions}

All Authors were involved in the preparation of this article. WG collected the data and wrote the article. SK, KT, YA, and TM performed the operation and designed the study. WG, SK, MS, and HT summarized the data and revised the article. KH and MO provided a substantial contribution to the study design, performed the operation, and revised the article. All Authors read and approved the final article.

\section{Acknowledgements}

The Authors thank Natsumi Okahara and Tomomi Okawa (Department of Breast and Endocrine Surgery, Osaka City University Graduate School of Medicine) for the helpful advice regarding data management. This study was funded by grants from the Japan Society for the Promotion of Science (KAKENHI, Nos. 19K18067, 20K08938, and 17K10559) to Shinichiro Kashiwagi. 


\section{References}

1 Towle MJ, Salvato KA, Budrow J, Wels BF, Kuznetsov G, Aalfs KK, Welsh S, Zheng W, Seletsky BM, Palme MH, Habgood GJ, Singer LA, Dipietro LV, Wang Y, Chen JJ, Quincy DA, Davis A, Yoshimatsu K, Kishi Y, Yu MJ and Littlefield BA: In vitro and in vivo anticancer activities of synthetic macrocyclic ketone analogues of halichondrin B. Cancer Res 61(3): 1013-1021, 2001. PMID: 11221827.

2 Kuznetsov G, Towle MJ, Cheng H, Kawamura T, TenDyke K, Liu D, Kishi Y, Yu MJ and Littlefield BA: Induction of morphological and biochemical apoptosis following prolonged mitotic blockage by halichondrin B macrocyclic ketone analog E7389. Cancer Res 64(16): 5760-5766, 2004. PMID: 15313917. DOI: $10.1158 / 0008-5472 . C A N-04-1169$

3 Jordan MA, Kamath K, Manna T, Okouneva T, Miller HP, Davis C, Littlefield BA and Wilson L: The primary antimitotic mechanism of action of the synthetic halichondrin E7389 is suppression of microtubule growth. Mol Cancer Ther 4(7): 10861095, 2005. PMID: 16020666. DOI: 10.1158/1535-7163.MCT04-0345

4 Funahashi Y, Okamoto K, Adachi Y, Semba T, Uesugi M, Ozawa Y, Tohyama O, Uehara T, Kimura T, Watanabe H, Asano M, Kawano S, Tizon X, McCracken PJ, Matsui J, Aoshima K, Nomoto $\mathrm{K}$ and Oda $\mathrm{Y}$ : Eribulin mesylate reduces tumor microenvironment abnormality by vascular remodeling in preclinical human breast cancer models. Cancer Sci 105(10): 1334-1342, 2014. PMID: 25060424. DOI: 10.1111/cas.12488

5 Yoshida T, Ozawa Y, Kimura T, Sato Y, Kuznetsov G, Xu S, Uesugi M, Agoulnik S, Taylor N, Funahashi Y and Matsui J: Eribulin mesilate suppresses experimental metastasis of breast cancer cells by reversing phenotype from epithelialmesenchymal transition (EMT) to mesenchymal-epithelial transition (MET) states. Br J Cancer 110(6): 1497-1505, 2014. PMID: 24569463. DOI: $10.1038 /$ bjc. 2014.80

6 Kashiwagi S, Asano Y, Goto W, Takada K, Takahashi K, Hatano T, Tanaka S, Takashima T, Tomita S, Motomura H, Ohsawa M, Hirakawa K and Ohira M: Mesenchymal-epithelial transition and tumor vascular remodeling in eribulin chemotherapy for breast cancer. Anticancer Res 38(1): 401-410, 2018. PMID: 29277801. DOI: 10.21873 /anticanres.12236

7 Goto W, Kashiwagi S, Asano Y, Takada K, Morisaki T, Fujita H, Takashima T, Ohsawa M, Hirakawa K and Ohira M: Eribulin promotes antitumor immune responses in patients with locally advanced or metastatic breast cancer. Anticancer Res 38(5): 29292938, 2018. PMID: 29715119. DOI: 10.21873/anticanres.12541

8 Kashiwagi S, Asano Y, Goto W, Takada K, Takahashi K, Noda S, Takashima T, Onoda N, Tomita S, Ohsawa M, Hirakawa K and Ohira M: Use of Tumor-infiltrating lymphocytes (TILs) to predict the treatment response to eribulin chemotherapy in breast cancer. PLoS One 12(2): e0170634, 2017. PMID: 28166544. DOI: 10.1371/journal.pone.0170634

9 Miyagawa Y, Araki K, Bun A, Ozawa H, Fujimoto Y, Higuchi T, Nishimukai A, Kira A, Imamura M, Takatsuka Y and Miyoshi Y: Significant association between low baseline neutrophil-tolymphocyte ratio and improved progression-free survival of patients with locally advanced or metastatic breast cancer treated with eribulin but not with nab-paclitaxel. Clin Breast Cancer 18(5): 400-409, 2018. PMID: 29605174. DOI: 10.1016/ j.clbc.2018.03.002
10 Miyoshi Y, Yoshimura Y, Saito K, Muramoto K, Sugawara M, Alexis K, Nomoto K, Nakamura S, Saeki T, Watanabe J, PerezGarcia JM and Cortes J: High absolute lymphocyte counts are associated with longer overall survival in patients with metastatic breast cancer treated with eribulin-but not with treatment of physician's choice-in the EMBRACE study. Breast Cancer 27(4): 706-715, 2020. PMID: 32133606. DOI: 10.1007/s12282-02001067-2

11 Greene FL and Sobin LH: A worldwide approach to the TNM staging system: collaborative efforts of the AJCC and UICC. J Surg Oncol 99(5): 269-272, 2009. PMID: 19170124. DOI: $10.1002 /$ jso. 21237

12 Cortes J, O'Shaughnessy J, Loesch D, Blum JL, Vahdat LT, Petrakova K, Chollet P, Manikas A, Diéras V, Delozier T, Vladimirov V, Cardoso F, Koh H, Bougnoux P, Dutcus CE, Seegobin S, Mir D, Meneses N, Wanders J, Twelves C and EMBRACE (Eisai Metastatic Breast Cancer Study Assessing Physician's Choice Versus E7389) investigators: Eribulin monotherapy versus treatment of physician's choice in patients with metastatic breast cancer (EMBRACE): a phase 3 open-label randomised study. Lancet 377(9769): 914-923, 2011. PMID: 21376385. DOI: 10.1016/S0140-6736(11)60070-6

13 Schöffski P, Chawla S, Maki RG, Italiano A, Gelderblom H, Choy E, Grignani G, Camargo V, Bauer S, Rha SY, Blay JY, Hohenberger P, D'Adamo D, Guo M, Chmielowski B, Le Cesne A, Demetri GD and Patel SR: Eribulin versus dacarbazine in previously treated patients with advanced liposarcoma or leiomyosarcoma: a randomised, open-label, multicentre, phase 3 trial. Lancet 387(10028): 1629-1637, 2016. PMID: 26874885. DOI: $10.1016 /$ S0 140-6736(15)01283-0

14 Eisenhauer EA, Therasse P, Bogaerts J, Schwartz LH, Sargent D, Ford R, Dancey J, Arbuck S, Gwyther S, Mooney M, Rubinstein L, Shankar L, Dodd L, Kaplan R, Lacombe D and Verweij J: New response evaluation criteria in solid tumours: revised RECIST guideline (version 1.1). Eur J Cancer 45(2): 228-247, 2009. PMID: 19097774. DOI: 10.1016/j.ejca.2008.10.026

15 Mantovani A, Allavena P, Sica A and Balkwill F: Cancer-related inflammation. Nature 454(7203): 436-444, 2008. PMID: 18650914. DOI: 10.1038 /nature07205

16 Lin EY and Pollard JW: Role of infiltrated leucocytes in tumour growth and spread. Br J Cancer 90(11): 2053-2058, 2004. PMID: 15164120. DOI: $10.1038 /$ sj.bjc. 6601705

17 Hoffmann TK, Dworacki G, Tsukihiro T, Meidenbauer N, Gooding W, Johnson JT and Whiteside TL: Spontaneous apoptosis of circulating $\mathrm{T}$ lymphocytes in patients with head and neck cancer and its clinical importance. Clin Cancer Res 8(8): 2553-2562, 2002. PMID: 12171883.

18 De Larco JE, Wuertz BR and Furcht LT: The potential role of neutrophils in promoting the metastatic phenotype of tumors releasing interleukin-8. Clin Cancer Res 10(15): 4895-4900, 2004. PMID: 15297389. DOI: 10.1158/1078-0432.CCR-030760

19 Cheung KL, Pinder SE, Paish C, Sadozye AH, Chan SY, Evans AJ, Blamey RW and Robertson JF: The role of blood tumor marker measurement (using a biochemical index score and cerbB2) in directing chemotherapy in metastatic breast cancer. Int J Biol Markers 15(3): 203-209, 2000. PMID: 11012094.

20 Kurebayashi J, Nishimura R, Tanaka K, Kohno N, Kurosumi M, Moriya T, Ogawa Y and Taguchi T: Significance of serum tumor markers in monitoring advanced breast cancer patients treated 
with systemic therapy: a prospective study. Breast Cancer 11(4): 389-395, 2004. PMID: 15604995. DOI: 10.1007/BF02968047

21 Khatcheressian JL, Hurley P, Bantug E, Esserman LJ, Grunfeld E, Halberg F, Hantel A, Henry NL, Muss HB, Smith TJ, Vogel VG, Wolff AC, Somerfield MR, Davidson NE and American Society of Clinical Oncology: Breast cancer follow-up and management after primary treatment: American Society of Clinical Oncology clinical practice guideline update. J Clin Oncol 31(7): 961-965, 2013. PMID: 23129741. DOI: 10.1200/JCO.2012.45.9859

22 Melichar B: Biomarkers in the treatment of cancer: opportunities and pitfalls. Clin Chem Lab Med 51(7): 1329-1333, 2013. PMID: 23729626. DOI: 10.1515/cclm-2013-0323
23 Kashiwagi S, Tsujio G, Asano Y, Goto W, Takada K, Takahashi K, Morisaki T, Fujita H, Takashima T, Tomita S, Ohsawa M, Hirakawa $\mathrm{K}$ and Ohira M: Study on the progression types of cancer in patients with breast cancer undergoing eribulin chemotherapy and tumor microenvironment. J Transl Med 16(1): 54, 2018. PMID: 29523158. DOI: 10.1186/s12967-018-1443-5

Received November 6, 2021

Revised December 30, 2021

Accepted January 7, 2022 